\title{
Glioblastoma Multiforme in a Patient with Isolated Hemimegalencephaly
}

\author{
Jan Chrastina ${ }^{1}$ Zdenek Novak ${ }^{1}$ Milan Brazdil ${ }^{1}$ Marketa Hermanova ${ }^{2}$
}

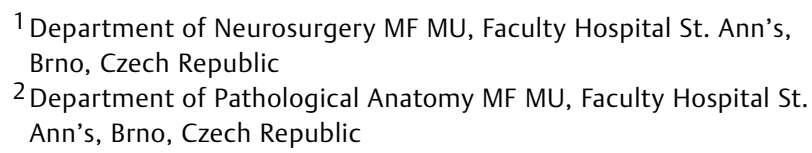
Brno, Czech Republic

2 Department of Pathological Anatomy MF MU, Faculty Hospital St. Ann's, Brno, Czech Republic

J Neurol Surg Rep 2015;76:e160-e163.

\begin{abstract}
Address for correspondence Jan Chrastina, MD, PhD, Department of Neurosurgery MF MU, Faculty Hospital St. Ann's, Pekarska 53, Brno 65691, Czech Republic (e-mail: jan.chrastina@fnusa.cz).
\end{abstract}

\author{
Abstract \\ Keywords \\ - malformations of \\ cortical development \\ - hemimegalencephaly \\ - epilepsy \\ - glioblastoma \\ multiforme
}

We present an exceptional case of a patient with hemimegalencephaly and secondary intractable epilepsy treated with vagus nerve stimulation (VNS) and subsequent glioblastoma development in the hemimegalencephalic hemisphere 6 years after surgery. VNS (at age 18 years) led to a $60 \%$ reduction of intractable seizures. However, symptoms of intracranial hypertension suddenly occurred 6 years after surgery. A computed tomography scan revealed a brain tumor in the hemimegalencephalic hemisphere. Pathologic examination confirmed glioblastoma multiforme. The genetic background of hemimegalencephaly is discussed here, with attention paid to the available data about the malignant transformation of malformations of cortical development (MCDs). The case points to the need for adequate clinical and radiologic follow-up care for patients with MCDs including hemimegalencephaly.

\section{Introduction}

Between $25 \%$ and $40 \%$ of childhood epilepsies are caused by malformations of cortical development (MCDs). ${ }^{1}$ MCDs result from abnormal neuronal and glial proliferation or differentiation (tuberous sclerosis, focal cortical dysplasia [FCD], hemimegalencephaly), anomalous neuronal migration (subcortical band heterotopia, periventricular nodular heterotopia), and abnormal cortical organization (polymicrogyria, schizencephaly). ${ }^{2}$ Hemimegalencephaly is a rare brain malformation (occurring in $0.1-0.3 \%$ of children with intractable epilepsy) caused by anomalous neuronal and glial proliferation or differentiation, with an abnormally enlarged and dysplastic hemisphere. ${ }^{3-5}$ Three forms of hemimegalencephaly are described: isolated (without hemicorporal hypertrophy or cutaneous or systemic involvement), syndromic (associated with other diseases), and total (enlargement of the ipsilateral brainstem and cerebellum). The main symptoms include epilepsy, psychomotor retardation, and contralateral hemiparesis.

received

October 27, 2014

accepted

March 12, 2015

published online

June 26, 2015
The main treatment goal in hemimegalencephalic patients is seizure control. Hemispherectomy or hemispheric disconnection is used in young children when other brain regions can take over some functions of the resected brain. ${ }^{6}$ If resective surgery is impossible, vagus nerve stimulation (VNS) can be used for seizure palliation. ${ }^{7}$

The etiology of hemimegalencephaly is not clear. Some authors consider abnormal neuroepithelial cell lineage as the primary cause; migratory disorders and cellular proliferation are secondary findings. ${ }^{8,9}$ In a study of resected hemimegalencephalic hemispheres, Lee et al found de novo somatic mutations in identified genes (phosphatidylinositol 3-kinase [PIK3CA], the protein AKT kinase [AKT3], and a mammalian target of rapamycin [mTOR]) that are known to be associated with malignant tumors (breast and pancreas). ${ }^{10}$ Because of the excessive neuronal and glial proliferation in hemimegalencephaly and also because of genetic changes associated with malignant tumors, an increased incidence of brain tumors might be expected in hemimegalencephalic patients. However, no data describing glioblastoma development in
License terms Stuttgart · New York

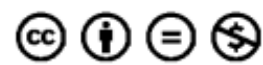


the hemimegalencephalic hemisphere have been published yet. We present an exceptional case of a patient with hemimegalencephaly who was followed throughout his life for intractable epilepsy treated with VNS, and with glioblastoma development in the hemimegalencephalic hemisphere 6 years after surgery.

\section{Case Description}

An 18-year-old man had been followed in the pediatric neurologic department since neonatal age for severe mental retardation and pharmacoresistant epilepsy. His family history was unremarkable, with no brain malformations or other neurologic diseases. The gestational period, delivery, and personal history were without problems. Neurologic examination revealed a slight left-sided hemiparesis, horizontal nystagmus, and convergent strabismus. There were several seizures types (myoclonic seizures, simple partial seizures with motor symptoms, complex partial seizures, and generalized tonic-clonic seizures). Scalp electroencephalogram revealed epileptic activity predominantly over the right hemisphere (gradual development of multifocal spike wave complexes with a tendency to generalize).

Repeated magnetic resonance imaging (MRI) studies before the subject was 10 years of age proved right hemisphere hemimegalencephaly. There were no findings suggesting syndromic hemimegalencephaly. At this point, the patient was referred for surgery, but resection was not indicated. The last follow-up MRI study (at age 17 years) confirmed stable findings.

After reaching adulthood (18 years), the patient was referred to the comprehensive epilepsy center for further treatment. Because of the intractability of his seizures, surgical treatment was reconsidered. A detailed MRI investigation confirmed the typical features of isolated hemimegalencephaly: increased cortical thickness, cortical-subcortical border blurring, irregular hyperintensity of hypertrophic white matter (advanced myelination) with the periventricular maximum also affecting the anterior callosal body, no detectable gray matter heterotopia, and no cerebellar or brainstem hypertrophy (-Fig. 1). Because of the patient's severe mental retardation, multifocal seizure origin, and adult age, resective surgery was contraindicated and VNS was implanted. Stimulation $(1.75 \mathrm{~mA}, 20 \mathrm{~Hz}, 250$ microseconds, 30 seconds on, 5 minutes off) led to a significant reduction in partial and generalized seizures ( $>60 \%$ ), and the patient became calmer and more communicative. The effect was stable during the entire follow-up period.

Six years after implantation, the patient sought an emergency evaluation after 3 weeks of a severe headache with vomiting that did not respond to analgesics or benzodiazepines, without seizure accumulation. The patient's neurologic status remained unchanged: discrete left-sided hemiparesis, horizontal nystagmus, and convergent strabismus. Computed tomography revealed an extensive brain tumor affecting the right frontal lobe with marked edema, midline shift, and irregular postcontrast enhancement; a high-grade glioma was suspected (-Fig. 2). MRI was not indicated because of the implanted VNS and the patient's mental retardation, restlessness, and anxiety. Only an incomplete tumor removal was possible because of the deep brain structure involvement and infiltrating growth. The patient awoke after this surgery without any new neurologic deficits.

Pathologic analysis confirmed a high-grade glial tumor with palisading necrosis, some large bizarre and multinucleated cells, and microvascular proliferation (-Fig. 3). Glial fibrillary acidic protein was expressed in most of the tumor cells, and diffuse nuclear p53 positivity was observed. Expression of isocitrate dehydrogenase 1 was examined immunohistochemically with negative immunostaining. The final diagnosis was glioblastoma multiforme.

The patient underwent radiotherapy with shortened fractionation to the residual tumor and tumor bed of $19 \times 2.5 \mathrm{~Gy}$. Concomitant chemotherapy was not indicated because of the risk of adverse effects. The seizure frequency remained stable after oncologic treatment, and stimulation parameters were not changed. The survival time was 28 months after tumor resection.

\section{Discussion}

Two important points should be emphasized when discussing this exceptional case. The first is the adequacy of the presurgical diagnostic work-up and the clinical course after VNS implantation, with attention paid to potential earlier tumor detection.

Repeated MRI studies confirmed isolated hemimegalencephaly with typical findings (marked hemispheric enlargement, moderate midline displacement, and moderate dilatation or reduction of the lateral ventricles) ${ }^{9,11}$ without any indications of brain tumor.

The literature provides only limited data about potential early markers of tumorous changes in hemimegalencephaly. Oikawa et al compared diffusion tensor imaging (DTI) results in three hemimegalencephalic patients (a 1-month-old, a 2month-old, and an 18-year-old adolescent) with findings of FCD, tuberous sclerosis, and gliomas. They proposed the use of DTI parameters, including fractional anisotropy maps and apparent diffusion coefficient, for evaluating the subcortical white matter adjacent to the blurred gray-white matter margins as a potentially valuable tool for distinguishing hemimegalencephaly from other diseases. However, the findings were not consistent, even in their small group. T2weighted white matter hyperintensity in the hemimegalencephalic hemisphere observed in the older adolescent patient was not found in the small children. ${ }^{12}$

Regarding the clinical course after VNS implantation and the possible warning symptoms of brain tumor, it is important to underline the good seizure outcome and psychological improvement of the patient, confirmed on multiple visits, before his sudden deterioration.

The second point concerns the causes of hemimegalencephaly and their potential association with malignant changes. A large variety of asymmetric brain disorders have a genetic background (e.g., Sturge-Weber syndrome, unilateral familial pachygyria, Lhermitte-Duclos disease, and progressive 


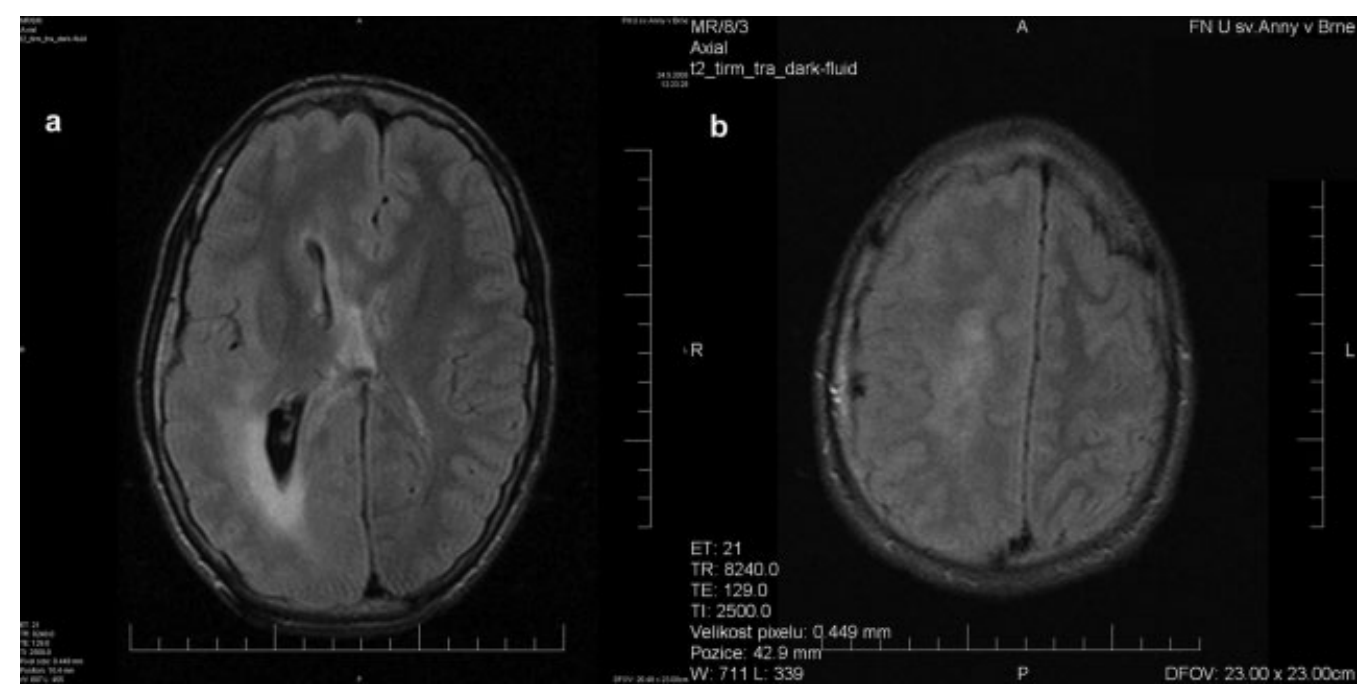

Fig. 1 Magnetic resonance imaging study (age 18 years). Isolated right hemisphere hemimegalencephaly. (A) Diffuse cortical thickening with some blurring of the cortical-subcortical border, irregular white matter hyperintensity, and right callosal body dysgenesis. (B) Extent of cortical involvement in the area corresponding to the brain tumor; note marked cranial asymmetry.

hemifacial atrophy). ${ }^{8,9}$ Genetic causes were identified in individual patients with syndromic hemimegalencephaly (Proteus syndrome). ${ }^{13}$ Recently Lee et al found de novo somatic gene mutations in the resected tissue from a subgroup of patients operated on for intractable epilepsy (hemispherectomy) caused by hemimegalencephaly. The mutations affected defined genes (PIK3CA, AKT3, and $m T O R$ ) regulating cell signaling in response to insulin and growth factors and influencing cell size, proliferation, differentiation, and apoptosis. The mutations were found in 8 to $40 \%$ of sequenced alleles in different brain regions. Therefore, hemimegalencephaly is a genetic mosaic disease caused by the functional increase in the signaling pathways of PIK3CA, AKT3, and $m T O R$. Although the mutations are known to be associated with malignant tumors (breast, pancreas), the presence of other mutations is needed for tumor development and dissemination. ${ }^{10,14}$ Neither phosphatase and tensin homolog (PTEN) gene mutation (Proteus syndrome) nor tuberous sclerosis complex genes (TSC1 or TSC2) were detected in the resected specimen of the hemimegalencephalic hemisphere. ${ }^{15}$ Some features of FCDs (cytomegaly, cortical stratification disruption, stem cell marker expression) can be related to $m T O R$ pathway hyperactivity. ${ }^{16}$ This pathway is a target of specific inhibitors (e.g., sirolimus and everolimus). ${ }^{17}$

Trophic factors may also play an important role in hemimegalencephaly development. ${ }^{8,9}$ Neural growth factor (NGF) is important for neuronal growth, differentiation, and survival. The increased tissue levels of NGF, numerous NGF receptor-positive cells, and NGF affinity for cerebral blood vessels and nerve fibers in the hemimegalencephalic hemisphere were confirmed, ${ }^{18}$ and

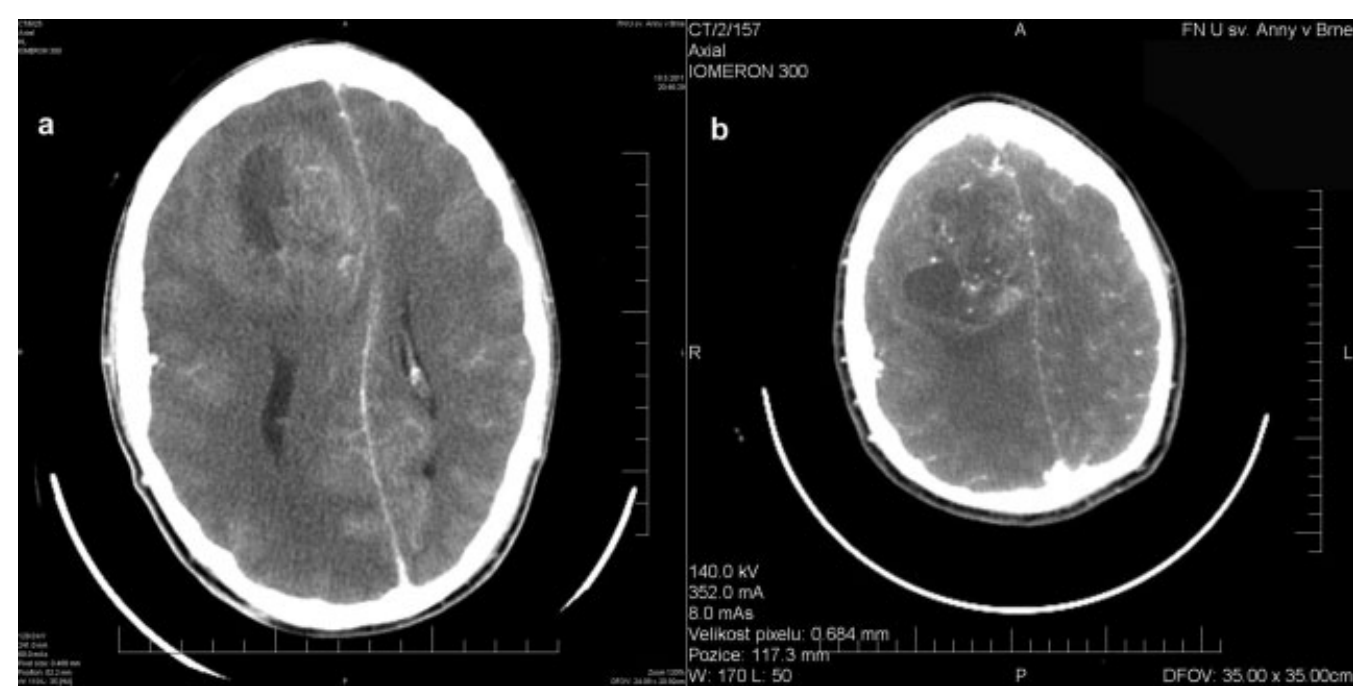

Fig. 2 Computed tomography image (age 24 years). Extensive right frontal tumor with postcontrast enhancement and central hypodensity. (A) Marked midline shift. (B) Extensive vascularity and cortical involvement. 


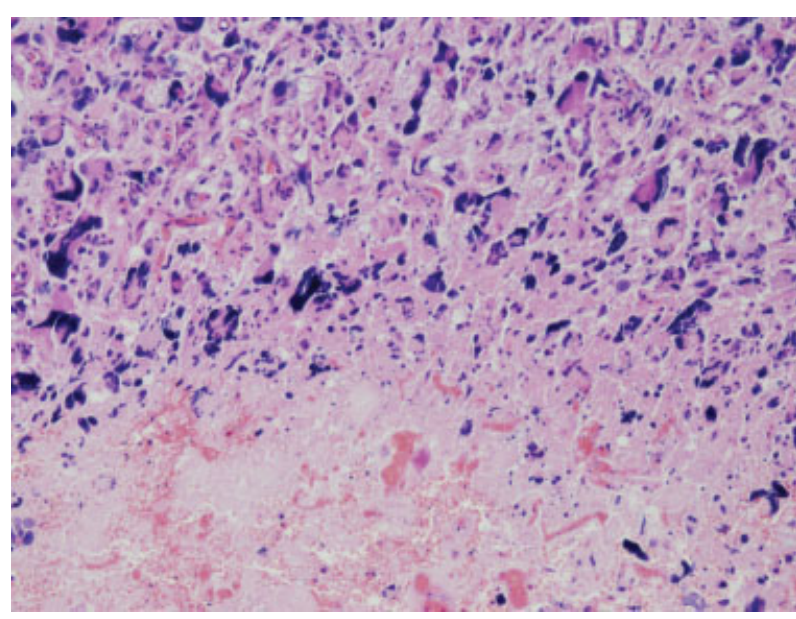

Fig. 3 Necrotizing glioblastoma with a high degree of anaplasia (original magnification $\times 100$; hematoxylin and eosin staining).

experimental studies proved a slight increase in tumor growth and tumor cell migration after NGF, as well as the role of NGF as a significant promotor of promigratory and proproliferative glioblastoma activities. ${ }^{19,20}$

The association of MCD and a malignant brain tumor is a rare event. ${ }^{21}$ Padmalatha et al described a young patient with tuberous sclerosis and glioblastoma. ${ }^{22}$ The development of glioblastoma after subependymal giant cell astrocytoma resection in a patient with tuberous sclerosis was published, but the tumor was probably radiation induced. ${ }^{23}$

Although exceptional, the association of MCD with a brain tumor should attract the attention of the treating physician to early identification of possible tumor signs. This requirement is necessary because VNS, which complicates a MRI study, is implanted in patients with unresectable extensive malformation and intractable epilepsy. The identification of another factor responsible for tumor formation with a background of these lesions or potential factors limiting tumor formation in a highrisk genetic background lesion is a problem for basic research.

\section{Conclusions}

The available data about the genetics of MCD, including hemimegalencephaly, suggest a potentially increased risk of malignant glioma growth in the malformed brain. Although exceptional, clinical articles confirming this risk in tuberous sclerosis patients and this case report, presenting so far unpublished glioblastoma formation in the affected hemisphere, indicate the need for meticulous clinical and radiologic follow-up care for MCD patients including those with hemimegalencephaly.

\section{References}

1 Kuzniecky RJ, Jackson GD. Magnetic Resonance in Epilepsy. New York, NY: Raven Press; 1995

2 Leventer RJ, Guerrini R, Dobyns WB. Malformations of cortical development and epilepsy. Dialogues Clin Neurosci 2008;10(1): 47-62
3 Di Rocco C, Battaglia D, Pietrini D, Piastra M, Massimi L. Hemimegalencephaly: clinical implications and surgical treatment. Childs Nerv Syst 2006;22(8):852-866

4 Manoranjan B, Provias JP. Hemimegalencephaly: a fetal case with neuropathological confirmation and review of the literature. Acta Neuropathol 2010;120(1):117-130

5 Perry MS, Duchowny M. Hemimegalencephaly. In: Shorvon S, Anderman F, Guerrini R, eds. The Causes of Epilepsy. Common and Uncommon Causes in Adults and Children. Cambridge, UK: Cambridge University Press; 2011:289-292

6 Wiebe S, Berg AT. Big epilepsy surgery for little people: what's the full story on hemispherectomy? Neurology 2013;80(3):232-233

7 Zamponi N, Rychlicki F, Corpaci L, Cesaroni E, Trignani R. Vagus nerve stimulation (VNS) is effective in treating catastrophic 1 epilepsy in very young children. Neurosurg Rev 2008;31(3): 291-297

8 Flores-Sarnat L. Hemimegalencephaly: part 1. Genetic, clinical, and imaging aspects. J Child Neurol 2002;17(5):373-384; discussion 384

9 Flores-Sarnat L, Sarnat HB, Dávila-Gutiérrez G, Alvarez A. Hemimegalencephaly: part 2 . Neuropathology suggests a disorder of cellular lineage. J Child Neurol 2003;18(11):776-785

10 Lee JH, Huynh M, Silhavy JL, et al. De novo somatic mutations in components of the PI3K-AKT3-mTOR pathway cause hemimegalencephaly. Nat Genet 2012;44(8):941-945

11 Battaglia D, Di Rocco C, Iuvone L, et al. Neuro-cognitive development and epilepsy outcome in children with surgically treated hemimegalencephaly. Neuropediatrics 1999;30(6):307-313

12 Oikawa T, Murata T, Tatewaki Y, et al. Can diffusion tensor imaging (DTI) be a useful tool in evaluating hemimegalencephaly? Poster C0962. Poster presented at: European Congress of Radiology 2014. Available at: http://dx.doi.org/10.1594/ecr2014/C-0962.

13 Lindhurst MJ, Sapp JC, Teer JK, et al. A mosaic activating mutation in AKT1 associated with the Proteus syndrome. N Engl J Med 2011; 365(7):611-619

14 Baek ST, Gibbs EM, Gleeson JG, Mathern GW. Hemimegalencephaly, a paradigm for somatic postzygotic neurodevelopmental disorders. Curr Opin Neurol 2013;26(2):122-127

15 Aronica E, Boer K, Baybis M, Yu J, Crino P. Co-expression of cyclin D1 and phosphorylated ribosomal S6 proteins in hemimegalencephaly. Acta Neuropathol 2007;114(3):287-293

16 Lim KC, Crino PB. Focal malformations of cortical development: new vistas for molecular pathogenesis. Neuroscience 2013; 252:262-276

17 Stefanaki K, Alexiou GA, Stefanaki C, Prodromou N. Tumors of central and peripheral nervous system associated with inherited genetic syndromes. Pediatr Neurosurg 2012;48(5):271-285

18 Antonelli A, Chiaretti A, Amendola T, Piastra M, Di Rocco C, Aloe L. Nerve growth factor and brain-derived neurotrophic factor in human paediatric hemimegalencephaly. Neuropediatrics 2004; 35(1):39-44

19 Brown MC, Staniszewska I, Lazarovici P, Tuszynski GP, Del Valle L, Marcinkiewicz C. Regulatory effect of nerve growth factor in alpha9beta1 integrin-dependent progression of glioblastoma. Neuro Oncol 2008;10(6):968-980

20 Engebraaten O, Bjerkvig R, Pedersen PH, Laerum OD. Effects of EGF, bFGF, NGF and PDGF(bb) on cell proliferative, migratory and invasive capacities of human brain-tumour biopsies in vitro. Int J Cancer 1993;53(2):209-214

21 Almairac F, Frenay M, Paquis P. Genetic diseases and glioblastomas [in French]. Neurochirurgie 2010;56(6):455-458

22 Padmalatha C, Harruff RC, Ganick D, Hafez GB. Glioblastoma multiforme with tuberous sclerosis. Report of a case. Arch Pathol Lab Med 1980;104(12):649-650

23 Matsumura H, Takimoto H, Shimada N, Hirata M, Ohnishi T, Hayakawa T. Glioblastoma following radiotherapy in a patient with tuberous sclerosis. Neurol Med Chir (Tokyo) 1998;38(5): 287-291 